\title{
Does the reason for discontinuation of a first TNF inhibitor influence the effectiveness of a second TNF inhibitor in axial spondyloarthritis? Results from the Swiss Clinical Quality Management Cohort
}

Adrian Ciurea ${ }^{1 *}$, Pascale Exer ${ }^{2}$, Ulrich Weber ${ }^{3,4}$, Giorgio Tamborrini ${ }^{5}$, Beate Steininger ${ }^{1}$, Rudolf O. Kissling ${ }^{6}$, Jürg Bernhard ${ }^{7}$, Almut Scherer ${ }^{8}$ and on behalf of the Rheumatologists of Swiss Clinical Quality Management Program for Axial Spondyloarthritis

\begin{abstract}
Background: With regard to switching tumor necrosis factor inhibitors (TNFi) in axial spondyloarthritis (axSpA), conflicting results have been reported as to whether the effectiveness of a second TNFi depends on the reason for discontinuation of the first TNFi.

Methods: Patients with a clinical diagnosis of axSpA starting a second TNFi in the Swiss Clinical Quality Management cohort were included. Effectiveness of treatment at 1 year, as well as drug survival, was compared between subgroups having discontinued the first TNFi because of lack of response, adverse events (AEs), or other reasons. Lack of response was further divided into primary or secondary lack of response (PLR or SLR, respectively), depending on whether the first TNFi was stopped before or after 6 months of treatment.

Results: Among 632 patients with axSpA, median survival of a second TNFi was 1.1 years after PLR and 3.8 years after $\operatorname{SLR}(p=0.003)$. At least moderate disease activity as defined by an Ankylosing Spondylitis Disease Activity Score using the erythrocyte sedimentation rate (ASDAS-ESR) $<2.1$ was achieved after 12 months by $11 \%, 39 \%, 26 \%$, and $39 \%$ of patients who discontinued their first TNFi because of PLR, SLR, AEs, and other reasons, respectively $(p=0.01)$. Only $4 \%$ of patients achieved an ASDAS-ESR inactive disease state after PLR, in comparison to $22 \%$ of those after SLR. Similar results were demonstrated in patients fulfilling the Assessment of SpondyloArthritis international Society classification criteria for axSpA ( $n=488$ ): ASDAS-ESR <2.1 was achieved after 12 months by $9 \%, 41 \%, 29 \%$, and $39 \%$ of patients who discontinued their first TNFi because of PLR, SLR, AEs, and other reasons, respectively $(p=0.01)$.
\end{abstract}

Conclusions: The effectiveness of a second TNFi is significantly impaired in patients with axSpA after PLR to a first TNFi compared with SLR.

Keywords: Axial spondyloarthritis, Tumor necrosis factor inhibitors, Switching, Drug retention

\footnotetext{
* Correspondence: adrian.ciurea@usz.ch

'Department of Rheumatology, University Hospital Zurich, Gloriastrasse 25,

$\mathrm{CH}-8091$ Zurich, Switzerland

Full list of author information is available at the end of the article
} 


\section{Background}

Although the use of tumor necrosis factor- $\alpha$ inhibitors (TNFi) has revolutionized the treatment of axial spondyloarthritis (axSpA), a significant proportion of patients do not adequately respond [1-5]. Young age, male sex, high baseline Bath Ankylosing Spondylitis Disease Activity Index (BASDAI), low baseline Bath Ankylosing Spondylitis Functional Index (BASFI), high baseline C-reactive protein (CRP), human leukocyte antigen B27 (HLA-B27) positivity, and the absence of enthesitis have been described as predictors of good response to TNFi $[6,7]$. We additionally identified smoking to be associated with a worse outcome following TNFi treatment in patients with axSpA [8]. Switching to an alternative TNFi appears to be associated with lower response and/or drug survival rates in patients with ankylosing spondylitis (AS) [9-24]. In patients with rheumatoid arthritis (RA), response to a second TNFi seemed better if the first TNFi was discontinued because of loss of efficacy or adverse events (AEs) in comparison to a primary lack of efficacy $[25,26]$. So far, no evidence for a differential response to a second TNFi in dependence on the reason for discontinuation of the first TNFi has been observed in axSpA. As new compounds with different modes of action are currently being tested in axSpA as potential alternatives to TNFi switching [27-29], we explored the effectiveness of switching TNFi in a large real-life observational axSpA cohort.

\section{Methods}

\section{Study population}

We conducted a longitudinal analysis of data collected annually from patients with a clinical diagnosis of axSpA, including AS, recruited in the ongoing Swiss Clinical Quality Management (SCQM) Cohort from January 2005 to September 2015 [30]. Clinical assessments included a physical examination (spinal and hip mobility according to the Bath Ankylosing Spondylitis Metrology Index, measurement of height and weight, presence of peripheral arthritis, dactylitis as well as enthesitis), laboratory tests (erythrocyte sedimentation rate [ESR] and CRP levels), data on treatment with nonsteroidal anti-inflammatory drugs (NSAIDs) as present or absent, and data on conventional and biologic disease-modifying drugs with dosage and start and stop dates [31]. The following reasons for drug discontinuation were specified in the database by the treating rheumatologist: insufficient effectiveness, AEs, remission, and other reasons. There was no further specification of the "other reasons" category of discontinuation in the SCQM questionnaire, but these reasons may be manifold, such as personal preference by the patient or physician, pregnancy, or elective surgery. As discontinuation due to remission was observed in only $1.9 \%$ of the patients, we pooled this category together with the "other reasons" category for discontinuation. Patient questionnaires included the BASDAI, the BASFI, smoking status (never, previous, or current), and the number of weekly exercise sessions.

\section{Inclusion criteria for the present study}

Patients with a clinical diagnosis of axSpA who had initiated a second TNFi after recruitment into the SCQMaxSpA cohort were included. Interruptions of treatment with the same TNFi were not counted as switches. Patients with overlapping TNFi courses or with an unclear start date were excluded. The study was approved by the ethics commission of the Canton of Zurich. Written informed consent was obtained from all patients.

\section{Outcomes}

The primary outcome was drug survival of a second TNFi in relation to the reason for discontinuation of the first TNFi. In the case of several reasons for drug discontinuation, the following hierarchy was implemented: lack of effect $>\mathrm{AE}>$ other reasons. Only the discontinuation reason highest in hierarchy was used. Lack of efficacy was further divided into primary lack of response (PLR) if the first TNFi was stopped within 6 months after start and in secondary lack of response (SLR) if the first TNFi was discontinued after a 6-month period. The Assessment of SpondyloArthritis international Society (ASAS) recommends assessment of response to treatment after at least 12 weeks [32]. We chose a cutoff of 6 months, however, as clinically relevant improvement may take longer than 3 months [33, 34]. Moreover, the treating rheumatologist has to apply for reimbursement for an alternative TNFi in Switzerland, which may delay the switching process for a couple of weeks. The co-primary outcome of interest was effectiveness of treatment, assessed as the proportion of patients reaching at least an Ankylosing Spondylitis Disease Activity Score (ASDAS) moderate disease activity state (ASDAS <2.1), an ASDAS inactive disease state (ASDAS <1.3), or the ASAS criteria for partial remission (ASAS-PR) at $12 \pm 3$ months [35, 36]. Achievement of ASDAS cutoffs is primarily presented using the ESR, as CRP levels are registered in SCQM Cohort with the respective reference level and not the detection level, thus impeding the recently proposed ASDAS-CRP imputation [37] in some patients. Results derived using ASDAS-CRP are presented in (see Additional file 2: Table S2) after assuming a constant number of 2 for CRP levels $<2$. Response was assessed in patients with available outcome values at 12 months. Patients with available outcome measures at this time point who had discontinued the first TNFi but had not started an alternative TNFi were considered nonresponders (response/tolerance analysis). Additionally, response was assessed only among 
patients still on treatment at $12 \pm 3$ months (perprotocol response analysis).

\section{Statistical analysis}

Baseline characteristics in terms of categorical variables were compared between patients starting a second TNFi after different reasons for discontinuation of the first TNFi using the $x^{2}$ test. For symmetrically distributed discrete or continuous variables, analysis of variance was used for testing whether the means in the different groups were equal. The Kruskal-Wallis test was used for data with skewed distribution. All tests were two-sided, with the significance level set at 0.05 .

Drug maintenance was described with Kaplan-Meier plots. The log-rank test was used for testing differences between groups shown in the plots. Multiple adjusted Cox proportional hazards models were set up to estimate a covariate-adjusted effect of the reason for discontinuation of the first TNFi on the drug maintenance of the second TNFi. Ongoing treatments were censored at the last visit in the cohort. The following covariates were used: sex, age, calendar year of switch (to account for the number of various anti-TNF agents available for switch), the individual anti-TNF agents, and the type of TNFi switch (monoclonal antibody [mAb] to $\mathrm{mAb}$ versus $\mathrm{mAb}$ to soluble receptor anti-TNF agent and vice versa). To assess the significance of differences in response rates after 1 year of treatment with the second TNFi, Fisher's exact test was used. R statistical software was used for all analyses.

\section{Results}

Patient disposition and baseline characteristics

A total of 686 patients with axSpA started treatment with a second TNFi after inclusion in the SCQM Cohort. A total of 54 patients lost to follow-up after the start of a second TNFi were excluded from the analyses. The baseline characteristics at the start of the second TNFi in these patients, stratified by the reason of discontinuation of the first TNFi (PLR in $23.1 \%$, SLR in $42.7 \%$, AEs in $19.8 \%$, other in $14.4 \%$ ), are shown in Table 1. There was an enrichment of patients with predictors of an impaired response to TNFi in the group having stopped the first TNFi because of PLR: higher proportion of HLA-B27 negativity and presence of enthesitis, higher BASFI, and higher proportions of smokers and of patients classified as having nonresponsive axSpA. Patients in the PLR group also displayed higher baseline BASDAI and ASDAS levels, and a higher percentage were treated with NSAIDs. A similar enrichment of patients with predictors of an unfavorable response, as well as of patients with a higher disease activity, was found in patients who met the ASAS classification criteria for axSpA $(n=488)$ (Table 2). The proportion of patients stopping their first TNFi because of PLR was similar in the groups

Table 1 Characteristics of patients with a clinical diagnosis of axial spondyloarthritis starting a second tumor necrosis factor inhibitor

\begin{tabular}{|c|c|c|c|c|c|c|}
\hline Parameter & Number of patients & $\operatorname{PLR}(n=146)$ & $\operatorname{SLR}(n=270)$ & $\mathrm{AE}(n=125)$ & Other $(n=91)$ & $p$ Value \\
\hline Male sex, $\%$ & 632 & 47.3 & 56.3 & 46.4 & 52.8 & 0.20 \\
\hline Age, years & 632 & $43.8 \pm 10.5$ & $44.4 \pm 11.1$ & $44.1 \pm 12.3$ & $45.0 \pm 13.1$ & 0.87 \\
\hline Radiographic axSpA, \% & 454 & 54.4 & 69.2 & 65.8 & 82.1 & 0.003 \\
\hline HLA-B27-positive, \% & 510 & 43.2 & 67.1 & 64.3 & 71.0 & $<0.001$ \\
\hline Elevated CRP, \% & 361 & 30.4 & 34.2 & 42.7 & 29.2 & 0.33 \\
\hline ASDAS-CRP & 316 & $3.4 \pm 0.8$ & $3.1 \pm 0.9$ & $3.0 \pm 1.1$ & $2.7 \pm 1.0$ & $<0.001$ \\
\hline ASDAS-ESR & 289 & $3.1 \pm 0.8$ & $2.8 \pm 0.8$ & $2.8 \pm 1.2$ & $2.6 \pm 1.2$ & 0.03 \\
\hline BASDAI & 348 & $6.0 \pm 1.8$ & $5.2 \pm 2.0$ & $4.9 \pm 2.3$ & $4.5 \pm 2.3$ & $<0.001$ \\
\hline BASFI & 345 & $4.6 \pm 2.5$ & $4.1 \pm 2.5$ & $3.7 \pm 2.5$ & $2.8 \pm 2.3$ & 0.001 \\
\hline BASMI & 288 & $2.1 \pm 1.9$ & $2.3 \pm 2.0$ & $2.4 \pm 2.1$ & $2.7 \pm 1.9$ & 0.36 \\
\hline Peripheral arthritis, \% & 629 & 41.4 & 37.0 & 31.7 & 30.8 & 0.26 \\
\hline Enthesitis heel, \% & 632 & 42.5 & 31.9 & 26.4 & 24.2 & 0.01 \\
\hline Smokers, \% & 602 & 67.6 & 57.2 & 69.0 & 50.0 & 0.01 \\
\hline DMARDs, \% & 632 & 21.2 & 24.1 & 12.0 & 24.2 & 0.03 \\
\hline NSAIDs, \% & 632 & 62.3 & 54.4 & 48.0 & 39.6 & 0.003 \\
\hline $\mathrm{BMI}, \mathrm{kg} / \mathrm{m}^{2}$ & 608 & $26.3 \pm 4.9$ & $25.6 \pm 4.9$ & $25.9 \pm 4.7$ & $25.7 \pm 4.7$ & 0.58 \\
\hline Years of education & 596 & $13.0 \pm 3.0$ & $13.5 \pm 3.0$ & $13.3 \pm 2.9$ & $14.1 \pm 3.2$ & 0.07 \\
\hline
\end{tabular}

axSpA axial spondyloarthritis, ASAS Assessment of SpondyloArthritis international Society, ASDAS-CRP Ankylosing Spondylitis Disease Activity Score using C-reactive protein, ASDAS-ESR Ankylosing Spondylitis Disease Activity Score using erythrocyte sedimentation rate, BASDAI Bath Ankylosing Spondylitis Disease Activity Index, BASFI Bath Ankylosing Spondylitis Functional Index, BASMI Bath Ankylosing Spondylitis Metrology Index, DMARDs disease-modifying antirheumatic drugs, NSAIDs nonsteroidal anti-inflammatory drugs, $B M I$ body mass index

Except where indicated otherwise, values for continuous variables are mean $( \pm S D)$ 
Table 2 Characteristics of patients fulfilling the ASAS axial spondyloarthritis classification criteria starting a second tumor necrosis factor inhibitor

\begin{tabular}{|c|c|c|c|c|c|c|}
\hline Parameter & Number of patients & $\operatorname{PLR}(n=110)$ & $\operatorname{SLR}(n=220)$ & $\operatorname{AE}(n=88)$ & Other $(n=70)$ & $p$ Value \\
\hline Male sex, \% & 488 & 47.3 & 60.9 & 53.4 & 55.7 & 0.12 \\
\hline Age, years & 488 & $42.8 \pm 10.2$ & $43.2 \pm 10.9$ & $42.3 \pm 11.8$ & $44.2 \pm 13.4$ & 0.77 \\
\hline Radiographic axSpA, \% & 388 & 66.7 & 77.4 & 82.5 & 87.9 & 0.02 \\
\hline HLA-B27-positive, \% & 442 & 51.6 & 73.7 & 75.0 & 78.3 & $<0.001$ \\
\hline Elevated CRP, \% & 285 & 34.8 & 37.0 & 52.0 & 30.8 & 0.15 \\
\hline ASDAS-CRP & 256 & $3.4 \pm 0.9$ & $3.1 \pm 0.9$ & $3.0 \pm 1.2$ & $2.7 \pm 1.1$ & 0.003 \\
\hline ASDAS-ESR & 233 & $3.2 \pm 0.8$ & $2.8 \pm 0.8$ & $2.8 \pm 1.2$ & $2.7 \pm 1.2$ & 0.08 \\
\hline BASDAI & 275 & $6.1 \pm 1.9$ & $5.1 \pm 2.0$ & $4.7 \pm 2.4$ & $4.6 \pm 2.3$ & $<0.001$ \\
\hline BASFI & 274 & $4.7 \pm 2.5$ & $4.1 \pm 2.5$ & $3.5 \pm 2.4$ & $3.0 \pm 2.2$ & 0.002 \\
\hline BASMI & 228 & $2.1 \pm 1.7$ & $2.3 \pm 2.0$ & $2.7 \pm 2.3$ & $2.9 \pm 2.0$ & 0.27 \\
\hline Peripheral arthritis, \% & 487 & 36.7 & 35.5 & 26.1 & 21.4 & 0.06 \\
\hline Enthesitis heel, \% & 488 & 40.0 & 33.2 & 26.1 & 22.9 & 0.06 \\
\hline Smokers, \% & 471 & 73.4 & 58.4 & 72.3 & 55.7 & 0.01 \\
\hline DMARDs, \% & 488 & 20.0 & 22.7 & 9.1 & 17.1 & 0.03 \\
\hline NSAIDs, \% & 488 & 62.7 & 54.5 & 50.0 & 35.7 & 0.01 \\
\hline BMl, $\mathrm{kg} / \mathrm{m}^{2}$ & 478 & $26.1 \pm 4.6$ & $25.7 \pm 4.8$ & $26.0 \pm 4.7$ & $26.2 \pm 4.8$ & 0.88 \\
\hline Years of education & 463 & $13.0 \pm 3.2$ & $13.5 \pm 3.0$ & $13.0 \pm 2.9$ & $13.9 \pm 3.2$ & 0.23 \\
\hline
\end{tabular}

axSpA axial spondyloarthritis, ASAS Assessment of SpondyloArthritis international Society, ASDAS-CRP Ankylosing Spondylitis Disease Activity Score using C-reactive protein, ASDAS-ESR Ankylosing Spondylitis Disease Activity Score using erythrocyte sedimentation rate, BASDAI Bath Ankylosing Spondylitis Disease Activity Index, BASFI Bath Ankylosing Spondylitis Functional Index, BASMI Bath Ankylosing Spondylitis Metrology Index, DMARDs disease-modifying antirheumatic drugs, NSAIDs nonsteroidal anti-inflammatory drugs, BMI body mass index

Except where indicated otherwise, values for continuous variables are mean $( \pm S D)$

with a clinical diagnosis of axSpA, those fulfilling the ASAS criteria, and those who met the modified New York criteria (23.1 \%, $22.5 \%$, and $20.0 \%$, respectively). A similar proportion of TNFi-treated patients in the PLR and SLR groups was concurrently treated with conventional disease-modifying antirheumatic drugs (Tables 1 and 2).

\section{Drug retention}

The median drug retention of the second TNFi was 2.29 years (95\% confidence interval $[\mathrm{CI}] 1.79-2.97)$ for all patients with axSpA and 2.61 years (95\% CI 2.05-3.28) in the subgroup fulfilling the ASAS axSpA classification criteria. Drug maintenance depending on the reason for discontinuation of the first TNFi is shown in Fig. 1 for all patients with a clinical diagnosis of axSpA and in Fig. 2 for patients fulfilling the ASAS axSpA classification criteria. Significant differences in retention rates were found between the four groups $(p=0.001)$, with the shortest drug survival observed after previous PLR. The median drug survival of a second TNFi was 1.06 years (95\% CI 0.75-1.96) after PLR and 3.76 years (95\% CI 3.12-4.28) after SLR $(p=0.003)$. This difference remained significant after adjustment for sex, age, the calendar year of switching (reflecting the number of available TNFi at each time point), and the type of TNFi switching ( $\mathrm{mAb}$ to $\mathrm{mAb}$ versus $\mathrm{mAb}$ to fusion protein anti-TNF agent and vice versa)

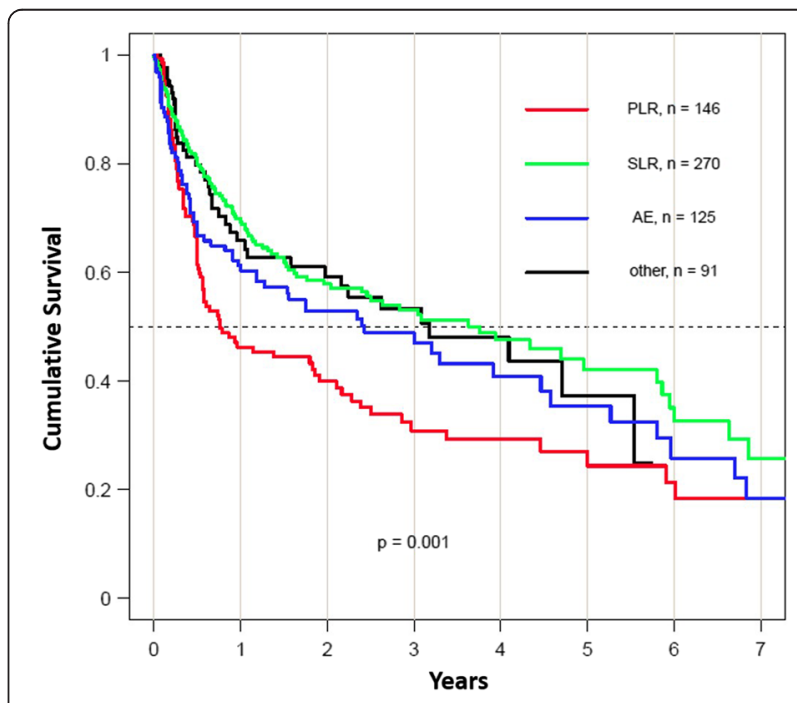

Fig. 1 Drug survival of the second tumor necrosis factor inhibitor (TNFi), stratified by the reason for discontinuation of the first TNFi, in patients with a clinical diagnosis of axial spondyloarthritis. Other refers to reason for discontinuation other than lack of effect or intolerance. AE adverse events, $P L R$ primary lack of response, SLR secondary lack of response 


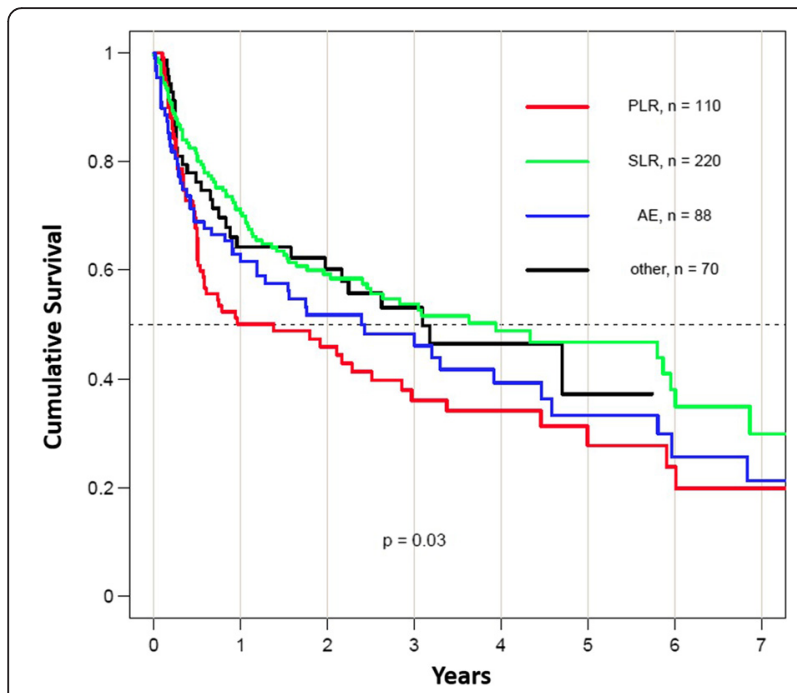

Fig. 2 Drug survival of the second tumor necrosis factor inhibitor (TNFi), stratified by the reason for discontinuation of the first TNFi, in patients fulfilling the Assessment of SpondyloArthritis international Society axial spondyloarthritis classification criteria. Other refers to reason for discontinuation other than lack of effect or intolerance. $A E$ adverse events, $P L R$ primary lack of response, SLR secondary lack of response

(Table 3). The hazard ratio for discontinuing the second TNFi after previous SLR in comparison to PLR was 0.56 (95\% CI $0.42-0.75, p<0.001)$ in all patients diagnosed as having axSpA and 0.58 (95\% CI 0.42-0.81, $p=0.002$ ) in those patients fulfilling the ASAS axSpA classification criteria. Similar results were found after replacing the type of TNFi switching by the various anti-TNF agents in the model (adalimumab, certolizumab, etanercept, golimumab, infliximab).

\section{Clinical response}

Response to treatment with a second TNFi was assessed in patients with available outcome values at
$12 \pm 3$ months (ASAS-PR $[n=227,36 \%]$ and ASDASESR $[n=184,29 \%])$. Patients with versus without follow-up at this time point did not differ with regard to important baseline disease characteristics (BASDAI, ASDAS, elevated CRP, HLA-B27, classification as AS, age, peripheral arthritis, enthesitis, smoking, education, reason for discontinuation of first TNFi, physical exercise, body mass index) (see Additional file 1: Table S1).

Response rates to a second TNFi differed significantly between the subgroups, based on the reasons for discontinuation of the first TNFi (Table 4). These were most impaired in the subgroup of patients having discontinued the first TNFi as a consequence of PLR, followed by AEs. At least moderate disease activity (defined by ASDAS-ESR <2.1) was reached by $11 \%, 26 \%$, and $39 \%$ of patients in the PLR, AE, and SLR groups, respectively. Only a negligible proportion of patients achieved ASASPR or ASDAS-ESR inactive disease state after PLR (2\% and $4 \%$ of patients, respectively), as opposed to $13 \%$ and $22 \%$, respectively, after SLR. Similar results were found in patients fulfilling the ASAS axSpA classification criteria (Table 4) and when using the CRP for ASDAS calculation (see Additional file 2: Table S2).

\section{Discussion}

Our TNFi switching study in axSpA, which to our knowledge is the largest so far, suggests that the reason for discontinuation of a first TNFi may affect the effectiveness of a second TNFi, as previously reported in RA $[25,26]$. Drug retention and treatment responses after switching to a second TNFi in axSpA were impaired in patients having discontinued the first TNFi due to primary lack of effectiveness in comparison to SLR. Earlier investigations had been hampered by the fact that it was often not possible to distinguish between these two reasons for drug discontinuation [18, 22]. As ASAS

Table 3 Cox models for drug retention of a second tumor necrosis factor inhibitor in patients having discontinued the first tumor necrosis factor inhibitor due to primary or secondary lack of response

\begin{tabular}{|c|c|c|c|c|c|c|}
\hline & \multicolumn{3}{|c|}{ Patients with a clinical diagnosis of axSpA ${ }^{a}$} & \multicolumn{3}{|c|}{ Patients fulfilling the ASAS axSpA classification criteria } \\
\hline & $\mathrm{HR}$ & $95 \% \mathrm{Cl}$ & $p$ Value & $H R$ & $95 \% \mathrm{Cl}$ & $p$ Value \\
\hline SLR vs. PLR & 0.56 & $0.42-0.75$ & $<0.001$ & 0.58 & $0.42-0.81$ & 0.002 \\
\hline Female vs. male & 0.94 & $0.71-1.24$ & 0.65 & 0.90 & $0.64-1.25$ & 0.53 \\
\hline Age & 1.00 & $0.99-1.02$ & 0.73 & 1.00 & $0.99-1.02$ & 0.93 \\
\hline Cept $\rightarrow \mathrm{mAb}$ & 1.11 & $0.79-1.55$ & 0.56 & 1.17 & $0.78-1.73$ & 0.45 \\
\hline $\mathrm{mAb} \rightarrow \mathrm{Cept}^{\mathrm{c}}$ & 1.11 & $0.80-1.56$ & 0.54 & 1.24 & $0.84-1.82$ & 0.28 \\
\hline 2007-2010 vs. before 2006 & 1.26 & $0.50-3.12$ & 0.63 & 1.18 & $0.47-2.96$ & 0.73 \\
\hline 2010-2015 vs. before 2006 & 1.58 & $0.63-3.99$ & 0.33 & 1.44 & $0.57-3.67$ & 0.44 \\
\hline
\end{tabular}

ASAS Assessment of SpondyloArthritis international Society, axSpA axial spondyloarthritis, Cept soluble receptor anti-tumor necrosis factor agent, mAb monoclonal antibody anti-tumor necrosis factor agent, $H R$ hazard ratio, $C l$ confidence Interval, PLR primary lack of response, SLR secondary lack of response

${ }^{\mathrm{a}}$ Total of 416 patients and 207 discontinuation events

${ }^{\text {b}}$ Total of 330 patients and 157 discontinuation events

Indicated switch type versus reference category $\mathrm{mAb} \rightarrow \mathrm{mAb}$ 
Table 4 Response rates after 1 year of treatment with a second tumor necrosis factor inhibitor, stratified by the reason of discontinuation of the first tumor necrosis factor inhibitor

\begin{tabular}{|c|c|c|c|c|c|c|c|c|c|}
\hline Response criterion & Analysis & Number of patients & All & PLR & SLR & $\mathrm{AE}$ & Other & $p$ Value $^{a}$ & $p$ Value $^{\mathrm{b}}$ \\
\hline \multicolumn{10}{|c|}{ Patients with a clinical diagnosis of axSpA } \\
\hline ASDAS-ESR $<2.1$ & Response/tolerance & 184 & 28.8 & 11.1 & 38.9 & 26.2 & 38.9 & 0.01 & 0.002 \\
\hline ASDAS-ESR $<2.1$ & Per protocol & 124 & 42.7 & 21.7 & 50.8 & 35.5 & 63.6 & 0.04 & 0.02 \\
\hline ASDAS-ESR $<1.3$ & Response/tolerance & 184 & 14.7 & 4.4 & 21.5 & 9.5 & 22.2 & 0.03 & 0.01 \\
\hline ASDAS-ESR $<1.3$ & Per protocol & 124 & 21.8 & 8.7 & 28.8 & 12.9 & 36.4 & 0.07 & 0.08 \\
\hline ASAS-PR & Response/tolerance & 227 & 11.0 & 2.0 & 12.5 & 13.7 & 16.7 & 0.08 & 0.04 \\
\hline ASAS-PR & Per protocol & 146 & 17.1 & 4.2 & 17.4 & 18.4 & 33.3 & 0.12 & 0.17 \\
\hline \multicolumn{10}{|c|}{ Patients fulfilling the ASAS axSpA classification } \\
\hline ASDAS-ESR $<2.1$ & Response/tolerance & 148 & 31.1 & 9.1 & 40.9 & 29.0 & 38.9 & 0.01 & 0.001 \\
\hline ASDAS-ESR $<2.1$ & Per protocol & 100 & 46.0 & 18.8 & 55.1 & 37.5 & 63.6 & 0.04 & 0.02 \\
\hline ASDAS-ESR $<1.3$ & Response/tolerance & 148 & 16.2 & 3.0 & 22.7 & 12.9 & 22.2 & 0.045 & 0.02 \\
\hline ASDAS-ESR $<1.3$ & Per protocol & 100 & 24.4 & 6.2 & 30.6 & 16.7 & 36.4 & 0.13 & 0.09 \\
\hline ASAS-PR & Response/tolerance & 179 & 11.7 & 0.0 & 12.5 & 16.7 & 18.5 & 0.03 & 0.03 \\
\hline ASAS-PR & Per protocol & 106 & 18.1 & 0.0 & 17.5 & 21.4 & 35.7 & 0.05 & 0.10 \\
\hline \multicolumn{10}{|c|}{$\begin{array}{l}\text { axSpA axial spondyloarthritis, ASDAS-CRP Ankylosing Spondylitis Disease Activity Score using C-reactive protein, ASDAS-ESR Ankylosing Spondylitis Disease Activity } \\
\text { Score using erythrocyte sedimentation rate, AE adverse events, PLR primary lack of response, SLR secondary lack of response } \\
\text { Other refers to reason of discontinuation other than lack of effect or intolerance. Response/tolerance refers to proportion of patients with a valid follow-up achieving the } \\
\text { respective response criterion (with patients having discontinued treatment being defined as nonresponders). Per protocol refers to proportion of patients achieving the } \\
\text { respective response criterion among those patients still receiving treatment } \\
\text { Except where indicated otherwise, values are percentages } \\
\text { ap Value overall } \\
{ }^{b} p \text { Value PLR vs. SLR }\end{array}$} \\
\hline
\end{tabular}

recommends assessment of treatment response after at least 12 weeks [32] but time to improvement may be longer than 3 months [33, 34], we have defined a discontinuation due to an insufficient effect after 6 months of treatment as being the consequence of a loss of efficacy. This cutoff allowed us to evaluate drug retention and response rates of the second TNFi. We found a difference of 2.7 years in median retention of the second TNFi between patients in the PLR and SLR groups. Moreover, an ASDAS-ESR inactive disease state was reached by only $4 \%$ of patients after previous PLR in comparison to $22 \%$ after SLR. Thus, PLR may identify a subgroup of patients in whom TNF probably does not play a major role in disease pathogenesis and amplification of inflammation. Whether these patients would experience a superior response to biologics with a different mode of action, as demonstrated for RA [38], remains to be established. An alternative, though mutually not exclusive, reason for impaired effectiveness of the second TNFi in the PLR group is a higher proportion of patients with predictors of an impaired response to anti-TNF agents [7] in our study (normal CRP, HLA-B27 negativity, high BASFI levels, and frequent enthesitis). Furthermore, misdiagnosis in some patients in the PLR group cannot be ruled out in the PLR group, given the low percentage of HLA-B27positive patients (43\%). We expected a lower proportion of PLR patients in the groups fulfilling the ASAS classification criteria or the modified New York classification criteria, which was not the case, however. Finally, even with a correct diagnosis of axSpA, patients may have additional reasons for back pain (e.g., degenerative spinal disease or fibromyalgia [39-41]), which may be misinterpreted as axSpA activity, prompting the initiation of antiTNF treatment. Reassessment of a diagnosis of axSpA and of musculoskeletal comorbidities in patients having experienced PLR to a first TNFi seems advisable before initiating a next biologic.

The results were very similar in patients with a clinical diagnosis of axSpA made by their treating rheumatologist and those fulfilling the ASAS classification criteria for axSpA. The presentation of patients with axSpA diagnosed on clinical grounds also allows comparison with TNFi switching in other observational cohorts $[18,22]$, where the proportion of patients fulfilling the modified New York criteria or the ASAS axSpA classification criteria was not reported. The median drug survival of a second TNFi was 2.3 years in SCQM and 1.6 years in the Danish DANBIO registry [22]. In DANBIO, the proportion of patients with a clinical diagnosis of AS treated for at least 2 years with a second TNFi who reached an ASDAS-CRP $<2.1$ was $37 \%$. We found a similar proportion of patients with axSpA (43\%) with an ASDAS-ESR $<2.1$ at 1 year of treatment with a second TNFi. Our result differed significantly in dependence on the reason for discontinuation of the first TNFi: only $22 \%$ after previous PLR, $36 \%$ after AEs and $51 \%$ after SLR. 
Over $75 \%$ of the patients in the SCQM Cohort met the ASAS classification criteria for axSpA. Patients fulfilling the ASAS criteria showed comparable response rates (with $19 \%, 38 \%$, and $55 \%$ reaching an ASDAS-ESR $<2.1$ in the PLR, AE, and SLR groups, respectively) to those of the patients in the entire cohort defined by the treating rheumatologists.

A limitation of our response analyses is that follow-up data of ASDAS at 12 months was available for only approximately one-third of patients. However, patients with versus without follow-up at this time point did not differ with regard to clinically relevant baseline disease characteristics. The limitation of incomplete follow-up is inherent to observational registries. In the NORDMARD registry [18], ASDAS responses were available in $25 \%$ of patients at 3 months and $29 \%$ at the last observation and in DANBIO in $45 \%$ of patients at 2 years [22]. There was no difference in response rates among the various anti-TNF agents used as second-line treatment in DANBIO, while drug survival of second treatment courses was longer in adalimumab-treated patients previously treated with infliximab [22]. In RA, drug survival of infliximab was shown to be inferior to adalimumab or etanercept [26], although other comparisons yielded a similar maintenance of various TNFi [42]. Moreover, the type of TNFi switch was shown to affect the effectiveness of a second TNFi in RA: Switching from an anti-TNF mAb to a soluble TNF receptor yielded better results than vice versa [26]. We were unable to confirm differences between individual anti-TNF agents or between various types of switching with regard to drug maintenance.

\section{Conclusions}

Our findings suggest that the effectiveness of a second TNFi is impaired in patients with axSpA who have experienced a PLR to a first TNFi during the first 6 months of treatment.

\section{Additional files}

Additional file 1: Table S1. Comparison of baseline characteristics of patients with and without a follow-up at 1 year of treatment. (DOC $38 \mathrm{~kb}$ )

Additional file 2: Table S2. ASDAS-CRP response rates after 1 year of treatment with a second TNFi, stratified by the reason for discontinuation of the first TNF inhibitor. (DOC $38 \mathrm{~kb}$ )

\footnotetext{
Abbreviations

AE: adverse event; AS: ankylosing spondylitis; ASAS: Assessment of SpondyloArthritis international Society; ASDAS: Ankylosing Spondylitis Disease Activity Score; axSpA: axial spondyloarthritis; BASDAl: Bath Ankylosing Spondylitis Disease Activity Index; BASFI: Bath Ankylosing Spondylitis Functional Index; BASMI: Bath Ankylosing Spondylitis Mobility Index; BMl: body mass index; Cl: confidence interval; CRP: C-reactive protein; DMARD: disease-modifying antirheumatic drug; ESR: erythrocyte sedimentation rate; HLA-B27: human leukocyte antigen B27; HR: hazard ratio; mAb: monoclonal antibody; NSAID: nonsteroidal anti-inflammatory drug; PLR: primary lack of
}

response; PR: partial remission; RA: rheumatoid arthritis; SCQM: Swiss Clinical Quality Management; SLR: secondary lack of response; TNFi: tumor necrosis factor inhibitor.

\section{Competing interests}

JB has received consulting fees from Merck Sharp \& Dohme, Pfizer, and Roche. AC has received consulting and/or speaking fees from AbbVie, Celgene, Eli Lilly, Janssen-Cilag, Merck Sharp \& Dohme, Novartis, Pfizer, and UCB. PE has received speaking fees from AbbVie and Merck Sharp \& Dome. UW has received speaking fees from AbbVie. The other authors declare that they have no competing interests.

\section{Authors' contributions}

AC made substantial contributions to study conception and design and to data acquisition, analysis, and interpretation, and drafted the manuscript. PE, GT, and JB made substantial contributions to data acquisition, analysis, and interpretation and revised the manuscript critically for important intellectual content. UW made substantial contributions to study design and to acquisition and interpretation of data, and helped to draft the manuscript. BS made substantial contributions to data acquisition, analysis, and interpretation and revised the manuscript. ROK made substantial contributions to study conception as well as to analysis and interpretation of data, and revised the manuscript. AS made substantial contributions to study design, performed the statistical analyses, and helped with data interpretation and drafting of the manuscript. All authors read and approved the final manuscript.

\section{Acknowledgments}

We thank all the rheumatologists and patients involved in the study for their participation, the members of the SCQM axSpA scientific board for contribution to pelvic $x$-ray scoring and helpful discussions, and the entire SCQM staff for data management and support. A list of rheumatology offices and hospitals that are contributing to the SCQM registries can be found at http://www.scam.ch/institutions. The SCQM Foundation is supported by the Swiss Society of Rheumatology and by AbbVie, Bristol-Myers Squibb, Merck Sharp \& Dohme, Pfizer, Roche, UCB, and Janssen and has received project-based financial support from the Arco Foundation, Switzerland, as well as from the Swiss Balgrist Society, Switzerland. This study was supported by an investigator-initiated study grant from AbbVie and by a grant from the "Stiftung für Rheumaforschung." The study sponsors had no role in the study design or in the collection, analysis, or interpretation of the data; the writing of the manuscript; or the decision to submit the manuscript for publication. Publication of this article was not contingent upon approval by the study sponsors.

\section{Author details}

${ }^{1}$ Department of Rheumatology, University Hospital Zurich, Gloriastrasse 25, CH-8091 Zurich, Switzerland. 'Private Rheumatology Practice, Basel, Switzerland. ${ }^{3}$ King Christian 10th Hospital for Rheumatic Diseases, Gråsten, Denmark. ${ }^{4}$ South Jutland Hospital, Denmark Institute of Regional Health Research, University of Southern Denmark, Odense, Denmark. ${ }^{5}$ Department of Rheumatology, Bethesda Hospital, Basel, Switzerland. ${ }^{6}$ Department of Rheumatology, Balgrist University Hospital, Zurich, Switzerland. ${ }^{7}$ Department of Rheumatology and Rehabilitation, Bürgerspital, Solothurn, Switzerland.

${ }^{8}$ Swiss Clinical Quality Management Foundation, Zurich, Switzerland.

Received: 19 January 2016 Accepted: 10 March 2016

Published online: 22 March 2016

\section{References}

1. van der Heijde D, Dijkmans B, Geusens P, Sieper J, DeWoody K, Williamson P, et al. Efficacy and safety of infliximab in patients with ankylosing spondylitis: results of a randomized, placebo-controlled trial (ASSERT). Arthritis Rheum. 2005:52:582-91.

2. Davis JC, van der Heijde D, Braun J, Dougados M, Cush J, Clegg D, et al. Sustained durability and tolerability of etanercept in ankylosing spondylitis for 96 weeks. Ann Rheum Dis. 2005;64:1557-62.

3. van der Heijde D, Kivitz A, Schiff MH, Sieper J, Dijkmans BA, Braun J, et al. Efficacy and safety of adalimumab in patients with ankylosing spondylitis: results of a multicenter, randomized, double-blind, placebo-controlled trial. Arthritis Rheum. 2006;54:2136-46. 
4. Inman RD, Davis Jr JC, van der Heijde D, Diekman L, Sieper J, Kim SI, et al. Efficacy and safety of golimumab in patients with ankylosing spondylitis: results of a randomized, double-blind, placebo-controlled, phase III trial. Arthritis Rheum. 2008;58:3402-12.

5. Landewé R, Braun J, Deodhar A, Dougados M, Maksymowych WP, Mease PJ, et al. Efficacy of certolizumab pegol on signs and symptoms of axial spondyloarthritis including ankylosing spondylitis: 24-week results of a double-blind randomised placebo-controlled phase 3 study. Ann Rheum Dis. 2014;73:39-47.

6. Maneiro JR, Souto A, Salgado E, Mera A, Gomez-Reino JJ. Predictors of response to TNF antagonists in patients with ankylosing spondylitis and psoriatic arthritis: systematic review and meta-analysis. RMD Open. 2015;1:e000017.

7. Vastesaeger $N$, van der Heijde D, Inman RD, Wang Y, Deodhar A, Hsu B, et al. Predicting the outcome of ankylosing spondylitis therapy. Ann Rheum Dis. 2011;70:973-81.

8. Ciurea A, Scherer A, Weber U, Exer P, Bernhard J, Tamborrini G, et al. Impaired response to treatment with tumour necrosis factor a inhibitors in smokers with axial spondyloarthritis. Ann Rheum Dis. 2015. doi:10.1136/annrheumdis-2013-205133.

9. Gomez-Reino J, Carmona L, BIOBADASER Group. Switching TNF antagonists in patients with chronic arthritis: an observational study of 488 patients over a four-year period. Arthritis Res Ther. 2006;8:R29.

10. Delaunay C, Farrenq V, Marini-Portugal A, Cohen JD, Chevalier X, Claudepierre $P$. Infliximab to etanercept switch in patients with spondyloarthropathies and psoriatic arthritis: preliminary data. J Rheumatol. 2005;32:2183-5.

11. Cantini F, Niccoli L, Benucci M, Chindamo D, Nannini C, Olivieri l, et al. Switching from infliximab to once-weekly administration of $50 \mathrm{mg}$ etanercept in resistant or intolerant patients with ankylosing spondylitis: results of a fifty-four-week study. Arthritis Rheum. 2006;55:812-6.

12. Conti F, Ceccarelli F, Marocchi E, Magrini L, Spinelli FR, Spadaro A, et al. Switching tumour necrosis factor a antagonists in patients with ankylosing spondylitis and psoriatic arthritis: an observational study over a 5-year period. Ann Rheum Dis. 2007;66:1393-7

13. Pradeep DJ, Keat AC, Gaffney K, Brooksby A, Leeder J, Harris C. Switching anti-TNF therapy in ankylosing spondylitis. Rheumatology (Oxford). 2008; $47: 1726-7$

14. Coates LC, Cawkwell LS, Ng NW, Bennett AN, Bryer DJ, Fraser AD, et al. Real life experience confirms sustained response to long-term biologics and switching in ankylosing spondylitis. Rheumatology (Oxford). 2008;47:897-900

15. Rudwaleit M, Van den Bosch F, Kron M, Kary S, Kupper H. Effectiveness and safety of adalimumab in patients with ankylosing spondylitis or psoriatic arthritis and history of anti-tumor necrosis factor therapy. Arthritis Res Ther. 2010;12:R117.

16. Spadaro A, Punzi L, Marchesoni A, Lubrano E, Mathieu A, Cantini F, et al. Switching from infliximab or etanercept to adalimumab in resistant or intolerant patients with spondyloarthritis: a 4-year study. Rheumatology (Oxford). 2010;49:1107-11.

17. Haberhauer G, Strehblow C, Fasching P. Observational study of switching anti-TNF agents in ankylosing spondylitis and psoriatic arthritis versus rheumatoid arthritis. Wien Med Wochenschr. 2010;160:220-4.

18. Lie E, van der Heijde D, Uhlig T, Mikkelsen K, Rodevand E, Koldingsnes W, et al. Effectiveness of switching between TNF inhibitors in ankylosing spondylitis: data from the NOR-DMARD register. Ann Rheum Dis. 2011;70:157-63.

19. Paccou J, Solau-Gervais E, Houvenagel E, Salleron J, Luraschi H, Philippe P, et al. Efficacy in current practice of switching between anti-tumour necrosis factor-a agents in spondyloarthropathies. Rheumatology (Oxford). 2011;50:714-20.

20. Plasencia C, Pascual-Salcedo D, Garcia-Carazo S, Lojo L, Nuno L, Villalba A, et al. The immunogenicity to the first anti-TNF therapy determines the outcome of switching to a second anti-TNF therapy in spondyloarthritis patients. Arthritis Res Ther. 2013;15:R79.

21. Spadaro A, Lubrano E, Marchesoni A, D'Angelo S, Ramonda R, Addimanda O, et al. Remission in ankylosing spondylitis treated with anti-TNF-a drugs: a national multicentre study. Rheumatology (Oxford). 2013;52:1914-9.

22. Glintborg B, Ostergaard M, Krogh NS, Tarp U, Manilo N, Loft AG, et al. Clinical response, drug survival and predictors thereof in 432 ankylosing spondylitis patients after switching tumour necrosis factor a inhibitor therapy: results from the Danish nationwide DANBIO registry. Ann Rheum Dis. 2013;72:1149-55.
23. Gulyas K, Bodnar N, Nagy Z, Szamosi S, Horvath A, Vancsa A, et al. Real-life experience with switching TNF-a inhibitors in ankylosing spondylitis. Eur J Health Econ. 2014;15 Suppl 1:S93-100.

24. Fabbroni M, Cantarini L, Caso F, Costa L, Pagano VA, Frediani B, et al. Drug retention rates and treatment discontinuation among anti-TNF-a agents in psoriatic arthritis and ankylosing spondylitis in clinical practice. Mediators Inflamm. 2014;2014:862969.

25. Remy A, Avouac J, Gossec L, Combe B. Clinical relevance of switching to a second tumour necrosis factor-a inhibitor after discontinuation of a first tumour necrosis factor-a inhibitor in rheumatoid arthritis: a systematic literature review and meta-analysis. Clin Exp Rheumatol. 2011;29:96-103.

26. Chatzidionysiou K, Askling J, Eriksson J, Kristensen LE, van Vollenhoven R, ARTIS group. Effectiveness of TNF inhibitor switch in RA: results from the national Swedish register. Ann Rheum Dis. 2015;74:890-6.

27. Baeten D, Baraliakos X, Braun J, Sieper J, Emery P, van der Heijde D, et al. Anti-interleukin-17A monoclonal antibody secukinumab in treatment of ankylosing spondylitis: a randomised, double-blind, placebo-controlled trial. Lancet. 2013;382:1705-13.

28. Poddubnyy D, Hermann KG, Callhoff J, Listing J, Sieper J. Ustekinumab for the treatment of patients with active ankylosing spondylitis: results of a 28-week, prospective, open-label, proof-of-concept study (TOPAS). Ann Rheum Dis. 2014;73:817-23.

29. Pathan E, Abraham S, Van Rossen E, Withrington R, Keat A, Charles PJ, et al. Efficacy and safety of apremilast, an oral phosphodiesterase 4 inhibitor, in ankylosing spondylitis. Ann Rheum Dis. 2013;72:1475-80.

30. Ciurea A, Scherer A, Exer P, Bernhard J, Dudler J, Beyeler B, et al. Tumor necrosis factor-a inhibition in radiographic and non-radiographic axial spondyloarthritis: results from a large observational cohort. Arthritis Rheum. 2013:65:3096-106

31. Sieper J, Rudwaleit M, Baraliakos X, Brandt J, Braun J, Burgos-Vargas R, et al. The Assessment of SpondyloArthritis international Society (ASAS) handbook: a guide to assess spondyloarthritis. Ann Rheum Dis. 2009;68 Suppl 2:ii1-44.

32. van der Heijde D, Sieper J, Maksymowych WP, Dougados M, Burgos-Vargas R, Landewé $\mathrm{R}$, et al. Update of the international ASAS recommendations for the use of anti-TNF agents in patients with axial spondyloarthritis. Ann Rheum Dis. 2011;70:905-8.

33. Braun J, van der Horst-Bruinsma IE, Huang F, Burgos-Vargas R, Vlahos B, Koenig AS, et al. Clinical efficacy and safety of etanercept versus sulfasalazine in patients with ankylosing spondylitis: a randomized, double-blind trial. Arthritis Rheum. 2011;63:1543-51.

34. Breban M, Vignon E, Claudepierre P, Devauchelle V, Wendling D, Lespessailles E, et al. Efficacy of infliximab in refractory ankylosing spondylitis: results of a sixmonth open-label study. Rheumatology (Oxford). 2002;41:1280-5.

35. Machado P, Landewé R, Lie E, Kvien TK, Braun J, Baker D, et al. Ankylosing Spondylitis Disease Activity Score (ASDAS): defining cut-off values for disease activity states and improvement scores. Ann Rheum Dis. 2011;70:47-53.

36. Anderson JJ, Baron G, van der Heijde D, Felson DT, Dougados M. Ankylosing spondylitis assessment group preliminary definition of short-term improvement in ankylosing spondylitis. Arthritis Rheum. 2001;44:1876-86.

37. Machado P, Navarro-Compan V, Landewé R, van Gaalen FA, Roux C, van der Heijde D. Calculating the Ankylosing Spondylitis Disease Activity Score if the conventional C-reactive protein level is below the limit of detection or if high-sensitivity c-reactive protein is used: an analysis in the DESIR cohort. Arthritis Rheumatol. 2015;67:408-13.

38. Finckh A, Ciurea A, Brulhart L, Moller B, Walker UA, Courvoisier D, et al. Which subgroup of patients with rheumatoid arthritis benefits from switching to rituximab versus alternative anti-tumour necrosis factor (TNF) agents after previous failure of an anti-TNF agent? Ann Rheum Dis. 2010;69:387-93.

39. Roussou E, Ciurtin C. Clinical overlap between fibromyalgia tender points and enthesitis sites in patients with spondyloarthritis who present with inflammatory back pain. Clin Exp Rheumatol. 2012;30(6 Suppl 74):24-30.

40. Almodovar R, Carmona L, Zarco P, Collantes E, Gonzalez C, Mulero J, et al. Fibromyalgia in patients with ankylosing spondylitis: prevalence and utility of the measures of activity, function and radiological damage. Clin Exp Rheumatol. 2010;28(6 Suppl 63):S33-9.

41. Salaffi F, De Angelis R, Carotti M, Gutierrez M, Sarzi-Puttini P, Atzeni F. Fibromyalgia in patients with axial spondyloarthritis: epidemiological profile and effect on measures of disease activity. Rheumatol Int. 2014;34:1103-10.

42. Fisher A, Bassett K, Wright JM, Brookhart MA, Freeman H, Dormuth CR. Comparative persistence of the TNF antagonists in rheumatoid arthritis - a population-based cohort study. PLoS One. 2014;9(8):e105193. 